\title{
Review
}

\section{Host plants and associated trophobionts of the weaver ants Oecophylla spp. (Hymenoptera: Formicidae)}

\author{
Grace T. Lim ${ }^{1,2, *}$, Laurence G. Kirton ${ }^{2}$, Scott M. Salom ${ }^{1}$, Loke T. Kok ${ }^{1}$, Richard D. Fell ${ }^{1}$ \\ and Douglas G. Pfeiffer ${ }^{1}$
}

Address: ${ }^{1}$ Entomology Department, Virginia Polytechnic Institute and State University, Blacksburg, VA, 24061, USA. ${ }^{2}$ Entomology Section, Forest Research Institute of Malaysia, 52109 Kepong, Selangor, Malaysia.

*Correspondence: Grace T. Lim. Fax. +603-62797575. Email: grace@frim.gov.my

Received: $\quad 5$ January 2008

Accepted: $\quad 30$ April 2008

doi: 10.1079/PAVSNNR20083035

The electronic version of this article is the definitive one. It is located here: http://www.cababstractsplus.org/cabreviews

(C) CAB International 2008 (Online ISSN 1749-8848)

\section{Abstract}

\begin{abstract}
Weaver ants (Oecophylla spp.) are often found on plants with insect symbionts (trophobionts), but the extent of such associations is not known. Examination of literature records of weaver ant host plants from 1900 to 2006 revealed that Oecophylla smaragdina, native to Asia, was recorded on 175 plant species in 46 families, with 28 associated trophobiont species in 7 families. Oecophylla longinoda, native to Africa, was recorded on 66 plant species in 34 families with 17 associated trophobiont species in 6 families. Both Oecophylla spp. shared host records on 17 economically important plant species. Such host plants could be used to augment establishment of weaver ants, facilitating their role as deterrents of phytophagous insect pests of economically important plants. $O$. smaragdina-tended trophobionts were recorded associating with the ants on several crops but rarely considered to be pests. Thus, the risk of trophobionts associated with Oecophylla ants being pests is considered minimal.
\end{abstract}

Keywords: Oecophylla smaragdina, Oecophylla longinoda, Host plant species, Trophobionts, Biological control

\section{Introduction}

The weaver ants, Oecophylla smaragdina Fabricius in Southeast Asia, Australia and the Western Pacific Islands [1] and Oecophylla longinoda Latrielle in Africa [2], have been studied for decades. 0 . smaragdina has been used by Chinese farmers to protect citrus crops since 304 A.D. [3]. Many studies on the bionomics of this ant genus [4-9] are from its application in the field. Farmer-friendly guides on applying $O$. smaragdina to fruit trees [10] and cashew [11] also exist that synthesize knowledge available on the ant in those habitats.

Oecophylla spp. form nests on a large number of host plant species. O. smaragdina is an effective biological control agent on host plants such as cashew [12], citrus [13] and mahogany [14] while $O$. longinoda has recently been reported to protect mango crops in Africa [15]. Host plants provide foliage the ants need to build nests with. $O$. smaragdina typically uses leaves of a certain 'normal' size that are not very waxy [8] and has been observed to favour certain plant species [5] but can construct nests with most leafy foliage. The ants can utilize many plants in a wide range of habitats [1]. Host plants also serve as an arboreal hunting ground provisioned with insects and other arthropods that the ant preys on, support trophobiont species (e.g. mealybugs and scale insects) that the ant tends for honeydew and nectar exudates that the ant consumes [16].

The references for host plants are fragmented, with the more extensive lists published by Way [4] for 0 . longinoda, and Begg [17], Peng et al. [6, 7] and Blüthgen et al. [16] for Australian 0 . smaragdina. Since the host plants in these lists and other references include those of economic value, all of which could derive benefit from weaver ant occupancy, the prospective applications for this biological control agent are considerable. This review brings together all host plant species records for Oecophylla spp. published since 1900. It may serve as a reference to screen for host plant species to which weaver ant protection could be applied, as well as hint towards possible 
Table 1 Records of Oecophylla smaragdina on confirmed and possible host plants, with associated trophobionts from a survey of the literature (1900-2006). Currently N accepted species and family names are used followed by names given in the original article within square brackets [ ], where different

Host plant species $^{1}$
1. Anacardiaceae
B1. Anacardium occidentale L.
B2. Buchanania arborescens (Blume) Blume
B3. Buchanania obovata Engl.
C1. Mangifera indica L.

C1. Mangifera indica $\mathrm{L}$.

A1. Pleiogynium timoriense (DC.) Leenh. [Pleiogynium timorense]

A2. Spondias dulcis Sol. ex Parkinson

2. Annonaceae

A3. Annona glabra L.

C2. Annona muricata L.

A4. Polyalthia holtzeana F. Muell.

A5. Polyalthia nitidissima (Dunal) Benth.

3. Apocynaceae

A6. Alstonia actinophylla (A. Cunn.) K Schum.

A7. Dyera costulata (Miq.) Hook. f.

Ichnocarpus frutescens $\mathrm{R}$. $\mathrm{Br}$.

A8. Melodinus australis Pierre

A9. Plumeria obtusa L.

Wrightia laevis subsp. millgar (Bailey) Ngan [ASCL]

A10. Wrightia pubescens $\mathrm{R}$. Br.

4. Arecaceae

Archontophoenix alexandrae (F. Muell.) F. Muell. Ex Benth.

D1. Areca catechu L.

A11. Carpentaria acuminata Becc.

A12. Caryota mitis Lour.

D2. Cocos nucifera L.

C. nucifera L. [PALM]

* Licuala ramsayi (F. Muell) Domin

Associated trophobiont

Egropa malayensis Dist.

Zesius chrysomallus Hubner

Arhopala micale Boisduval

Arhopala centaurus Fabricius

Mealybug sp.

Scale insect $\mathrm{sp}$

Scale insect $\mathrm{sp}$

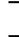

Mealybug sp.

Scale insect $\mathrm{sp}$.

Milviscutulus sp.

Cerataphis lataniae Boisduval

Icerya aegyptiaca Doug

$-$

Laingiococcus painei Laing

Scale insect sp.

Maculicoccus malaitensis (Cockerell)

Mutabilicoccus simmondsi (Laing) comb. nov.

Mealybug sp.

Scale insect $\mathrm{sp}$.

-

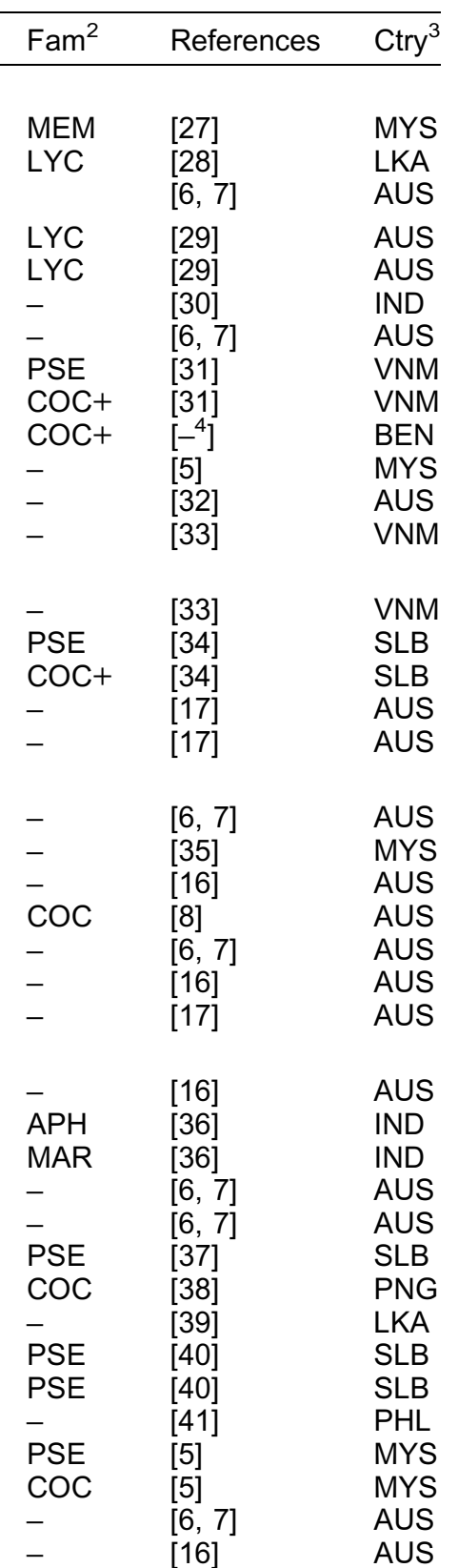


A13. Livistona humilis $\mathrm{R}$. Br.

Normanbya normanbyi (W. Hill) L.H. Bailey [PALM]

5. Bignoniaceae

Neosepicaea jucunda (F. Muell.) Steenis

A14. Tabebuia pallida (Lindl.) Miers

6. Boraginaceae

A15. Cordia curassavica (Jacq.) Roem. \& Schult

B4. Cordia dichotoma G. Forst.

7. Burseraceae

A16. Canarium album Raeusch

A17. Canarium australianum F. Muell.

8. Cannabaceae

Aphananthe philippinensis Planch. [ULM+]

A18. Celtis philippensis Blanco [Celtis philippinensis]

9. Capparaceae

A19. Capparis sepiaria L.

10. Caricaceae

A20. Carica papaya L.

Casuarinaceae

$$
\text { Casuarina sp. }
$$

11. Chrysobalanaceae

B5. Maranthes corymbosa Blume

B6. Parinari nonda Benth.

12. Clusiaceae

B7. Calophyllum inophyllum $\mathrm{L}$.

C. inophyllum L. [Colophylum inophilum] [GUTT]

A21. Garcinia mangostana L.

13. Combretaceae
B8. Lumnitzera racemosa Willd
B9. Terminalia arjuna (Roxb. ex DC.) Wight \& Arn.

D3. Terminalia catappa L.

A22. Terminalia grandiflora Benth. [Terminalia grandeflora]

B10. Terminalia melanocarpa $\mathrm{F}$. Muell.

B11. Terminalia muelleri Benth.

B12. Terminalia sericocarpa F. Muell.

T. sericocarpa F. Muell. [Terminalia seriocarpa] Terminalia spp.

14. Convolvulaceae

Ipomoea indica (Burm.) Merr.

13. Merremia peltata Merrill

15. Dipterocarpaceae

B14. Balanocarpus heimii King

A23. Shorea talura Roxb.

AUS

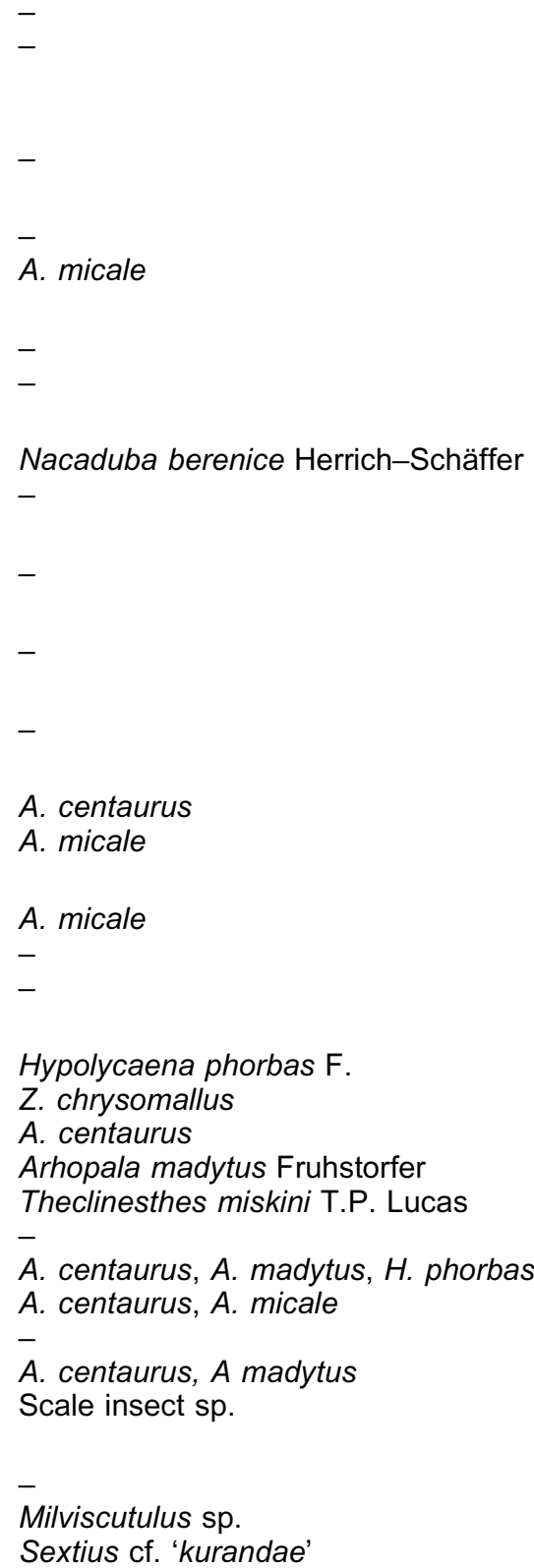

$-$

A. micale

Nacaduba berenice Herrich-Schäffer

$-$

\section{A. centaurus}

A. micale

A. micale

$-$

Hypolycaena phorbas $\mathrm{F}$

Z. chrysomallus

A. centaurus

Arhopala madytus Fruhstorfer

Theclinesthes miskini T.P. Lucas

A. centaurus, A. madytus, $H$. phorbas A. centaurus, A. micale

A. centaurus, A madytus

Scale insect $\mathrm{sp}$.

iscutulus sp.

Sextius cf. 'kurandae'

Anthene emolus goberus Fruhstorfer Coccus sp. [Lecanium sp.]
- [42] MYS

[29]

[3]

AUS

CHN

AUS

LYC [29]

AUS

AUS

- [17]

- [43]

AUS

MYS

$-\quad[32]$

AUS

$\begin{array}{ll}\text { LYC } & \text { [29] } \\ \text { LYC } & \text { [29] }\end{array}$

AUS

AUS

LYC

$[29]$
$[6,7]$

-

44]

LYC

[29]

[28]

LYC [29]

LYC

LYC

LYC

$\mathrm{COC}+$

[29]

$[6,7]$

[29]

[17]

[29]

COC [16]

MEM [8]

AUS

AUS

AUS

AUS

LKA

AUS

AUS

AUS

AUS

AUS

AUS

AUS

AUS

AUS

AUS

AUS

AUS

$\begin{array}{ll}\mathrm{LYC} & {[35]} \\ \mathrm{COC} & {[46]}\end{array}$ 
16. Ebenaceae

A24. Diospyros calycantha O. Schwarz

Elaeocarpus angustifolius Blume

\section{Euphorbiaceae}

A25. Croton schultzii Benth.

A26. Croton verreauxii Baill.

$$
\text { Endospermum myrmecophilum L.S. Sm. }
$$

B15. Hevea brasiliensis (Willd. ex A.H.L. Jussieu) Müll. Arg

Homalanthus novoguineensis (Warb.) K. Schum.

Macaranga involucrata subsp. mallotoides (F. Muell.) L.M. Perry Mallotus mollissimus (Geiseler) Airy Shaw

\section{Fabaceae}

A27. Abrus precatorius $L$.

* Acacia acradenia F. Muell. [MIMO]

Acacia alexandri Maslin [MIMO]

Acacia anceps DC. [MIMO]

A28. Acacia aulacocarpa A. Cunn. ex Benth. [MIMO]

B16. Acacia auriculiformis A. Cunn. ex Benth.

$$
\text { A. auriculiformis A. Cunn. ex Benth. [MIMO] }
$$

* Acacia crassicarpa A. Cunn. ex Benth. [MIMO] Acacia flavescens A. Cunn. ex Benth. [MIMO]

Acacia harpophylla F. Muell. ex Benth. [MIMO]

A29. Acacia hemignosta A. Cunn. ex Benth. [MIMO]

A30. Acacia holosericea A. Cunn. ex G. Don [MIMO]

B17. Acacia mangium Willd. [MIMO]

Acacia neriifolia A. Cunn. ex Benth. [MIMO]

Acacia polystachya A. Cunn. ex Benth. [MIMO]

Acacia pycnantha Benth. [MIMO]

Acacia salicina Lindl. [MIMO]

Acacia saligna (Labill.) H. L. Wendl. [MIMO]

Acacia tetragonophylla F. Muell. [MIMO]

Acacia victoriae Benth. [MIMO]

B18. Bauhinia monandra Kurz [LEGU]

Caesalpinia bonduc (L.) Roxb. [CAES]

Caesalpinia crista L. [CAES]

Caesalpinia mexicana A. Gray [CAES]

A31. Caesalpinia pulcherrima (L.) Sw. [CAES]

B19. Caesalpinia traceyi L. Pedley [CAES] 
* Cajanus reticulatus (Aiton) F. Muell.

Calliandra houstoniana (Mill.) Standl. [MIMO]

Calliandra surinamensis Benth. [MIMO]

A32. Canavalia rosea (Sw.) DC. [Canavalia maritima]

B20. Cassia auriculata L. [CAES]

B21. Cassia fistula L.

C. fistula L. [CAES]

B22. Castanospermum australe A. Cunn. \& C. Fraser ex Hook. Cathormion umbellatum (Vahl) Kosterm.

B23. Dalbergia sissoo Roxb. ex DC.

D4. Delonix regia (Bojer ex Hook.) Raf. [CAES]

Dendrolobium umbellatum (L.) Benth.

B24. Entada phaseoloides Merrill [MIMO]

A33. Erythrophleum chlorostachys (F. Muell.) Baill. [CAES]

B25. Inocarpus fagifer (Parkinson) Fosberg [Inocarpus edulis]

B26. Millettia pinnata (L.) Panigrahi

M. pinnata (L.) Panigrahi [Pongamia pinnata]

Paraserianthes lophanta (Willd.) I.C. Nielsen [MIMO]

B27. Pueraria phaseoloides (Roxb.) Bth.

B28. Saraca thaipingensis Cantley ex Prain

S. thaipingensis Cantley ex Prain [CAES]

B29. Schotia brachypetala Sond.

B30. Senna alata (L.) Roxb.

Senna auriculata (L.) Roxb.

Senna gaudichaudii (Hook. \& Arn.) H.S. Irwin \& Barneby

[Senna retusa]

Senna surattensis (Burm. f.) H.S. Irwin \& Barneby

Sesbania cannabina (Retz.) Pers.

Sesbania javanica Miq. [Sesbania javanicus]

Sesbania sp.

19. Flagellariaceae

B31. Flagellaria indica Linn

20. Lamiaceae

B32. Clerodendrum floribundum (R. Br.) [VERB]

B33. Clerodendrum inerme (L.) Gaertn. [VERB]
T. miskini

A. lycaenoides

A. lycaenoides

Z. chrysomallus

Z. chrysomallus
A. lycaenoides

Anthene lycaenoides godeffroyi (Semper)

H. phorbas

A. lycaenoides

Anthene seltuttus Röber

H. phorbas

T. miskini

Coccus hesperidum L. [Lecanium hesperidum]

Hilda bengalensis

Icerya sp.

Oxyrhachis tarandus $\mathrm{F}$.

A. seltutus

A. lycaenoides

Coccus sp.

Planococcus citri (Risso)

Sextius cf. 'kurandae'

M. malaitensis, Paraputo leveri (Green) (comb. nov.)

A. seltutus

A. lycaenoides

T. miskini

Catochrysops panormus Felder

Rapala pheretima Hewitson

A. seltutus

A. emolus goberus

A. seltutus

H. phorbas

A. lycaenoides

A. lycaenoides

A. lycaenoides

T. miskini

T. miskini

T. miskin

LYC [29] AUS

$\begin{array}{lll}\text { LYC } & {[29]} & \text { AUS } \\ \text { LYC } & {[29]} & \text { AUS }\end{array}$

[17] AUS

[YC [28] LKA

[29] AUS

$\begin{array}{lll}\text { LYC } & {[49]} & \text { AUS } \\ \text { LYC } & {[29]} & \text { AUS }\end{array}$

$\begin{array}{lll}\text { LYC } & {[29]} & \text { AUS } \\ \text { LYC } & {[29]} & \text { AUS }\end{array}$

LYC [49] AUS

LYC [29] AUS

LYC [29] AUS

COC [30] IND

TET [30]

MAR [30]

MEM [30]

LYC [29]

[29]

$\begin{array}{ll}\text { COC } & {[8]} \\ \text { PSE } & {[8]}\end{array}$

MEM

PSE

LYC

LYC

LYC

LYC

LYC

LYC

LYC

LYC

LYC

LYC

LYC

LYC

LYC

LYC

LYC

IND

IND

H. phorbas

LYC

Scale insect $\mathrm{sp}$.

$\mathrm{COC}+$

LYC

[29]

AUS

AUS

AUS

AUS

AUS

AUS

AUS

LYC

LYC

[29]
[29]

AUS

H. phorbas 


Associated trophobiont

$\begin{array}{ll}\text { Fam }^{2} & \text { R } \\ \text { LYC } & {[29]} \\ \text { MEM } & {[1} \\ - & {[1} \\ \text { LYC } & {[29} \\ \text { LYC } & {[29} \\ - & {[13} \\ - & {[30} \\ \text { PSE } & {[48} \\ - & {[17}\end{array}$

* Clerodendrum sp. [VERB]

* Clerodendrum sublimis

Clerodendrum tracyanum (F. Muell.) F. Muell. Ex Benth

A. lycaenoides

Austrotartessus spp.

B34. Faradaya splendida F. Muell. [VERB]

A. micale, $H$. phorbas

A34. Premna integrifolia L.

D5. Tectona grandis L. $\mathrm{f}$.

T. grandis L. f. [VERB]

A35. Vitex acuminata $\mathrm{R}$. $\mathrm{Br}$.

21. Lauraceae

B35. Cryptocarya hypospodia F. Muell.

A. lycaenoides

P. lilacinus

A. seltutus, A. micale

Toxoptera aurantii (Boyer de Fonscolombe)

Unidentified immatures

Cryptocarya murrayi F. Muell.

B36. Endiandra microneura C.T. White

B37. Endiandra cf. monothyra B.P.M. Hyland

A36. Litsea glutinosa (Lour.) C.B. Rob.

C3. Persea americana Mill.

22. Lecythidaceae

B38. Planchonia careya (F. Muell.) R. Knuth

23. Loganiaceae

A37. Strychnos lucida R. Br.

24. Loranthaceae

B39. Dendrophthoe vitellina (F. Muell.) Tiegh. Loranthus sp.

25. Lythraceae

B40. Lagerstroemia speciosa (L.) Pers.

A38. Sonneratia caseolaris (L.) Engl.

A. centaurus group

Coccus sp., Milviscutulus sp.

T. aurantii

Unidentified

Coccus sp., Milviscutulus sp.

Not collected

Sextius cf. 'kurandae'

H. phorbas

Ctry

AUS

AUS

[16] AUS

29] AUS

29] AUS

VNM

VNM
IND
PHL

AUS

LYC [29] AUS

$\begin{array}{lll} & & \\ \mathrm{APH} & {[29]} & \text { AUS }\end{array}$

COC [8] AUS

YC [16] AUS

[8] AUS

[8] AUS

[8] AUS

[8] AUS

$\begin{array}{lll}\mathrm{COC} & {[8]} & \text { AUS } \\ \mathrm{YYC} & {[8]} & \text { AUS }\end{array}$

MEM [8] [17] AUS

[17]

- $[6,7]$

LYC $[6,7] \quad$ AUS

[17]

AUS

A. centaurus, $H$. phorbas

P. citri [Dactylopius citri (Pseudococcus citri)]

Saissetia coffeae (Walker) [Lecanium hemisphaericum

(Saissetia hemisphaericum)]

Z. chrysomallus

[29] AUS

[51] IDN

COC [51] IDN

A. seltutus, A. centaurus, A. micale

LYC [28]

LKA

Coccid sp.

LYC [29] AUS

Rhyssopterys timoriensis (DC.)

Blume ex A.H.L. Jussieu [Rhyssopterys timorensis]

A. lycaenoides

LYC [29]

AUS

6. Malvacea

A39. Argyrodendron peralatum (F.M. Bailey) Edlin ex J.H. Boas [STER]

A40. Bombax ceiba L.

B41. Brachychiton acerifolius (A. Cunn. ex G. Don) Macarthur [STER]

A41. Ceiba pentandra (L.) Gaertn.

A. seltutus

$\begin{array}{ll}- & {[8]} \\ - & {[17]} \\ \text { LYC } & {[29]} \\ - & {[13]}\end{array}$

AUS

13] 
B42. Heritiera littoralis Aiton [STER]

A42. Sterculia quadrifida $\mathrm{R}$. Br.

B43. Talipariti tiliaceum (L.) Fryxell [Hibiscus tiliaceus]

D6. Theobroma cacao L. [STER]

Melastomataceae

Memecylon umbellatum Kostel

27. Meliaceae

Dysoxylum mollissimum subsp. molle (Miq.) D.J. Mabberley

Dysoxylum papuanum Mabb.

Dysoxylum pettigrewianum F.M. Bailey

A43. Khaya ivorensis A. Chevalier

Toona ciliata M. Roem

A44. Vavaea australiana S.T. Blake

B44. Xylocarpus moluccensis (Lam.) M. Roem. Xylocarpus sp.

28. Menispermaceae

Pachygone longifolia F.M.Bailey

A45. Pachygone ovata (Poir.) Hook. f. \& Thomson

A46. Stephania japonica Miers

29. Moraceae

C4. Artocarpus heterophyllus Lam.

A47. Ficus madurensis Miq

A48. Ficus opposita Miq.

A49. Ficus pantoniana King

B45. Ficus religiosa L.

\section{B46. Ficus septica Burm. f} Ficus $\mathrm{sp}$.

A50. Malaisia scandens (Lour.) Planch.

30. Myristicaceae

B47. Myristica insipida $\mathrm{R}$. Br.

31. Myrsinaceae

B48. Aegiceras corniculatum (L.) Blanco

* Ardisia pachyrrachis (F.Muell.) F.M. Bailey Embelia caulialata S.T.Reynolds

32. Myrtaceae

A51. Acmena graveolens L.S. Smith Acmena sp.

B49. Corymbia intermedia (R.T. Baker) K.D. Hill \& L.A.S. Johnson
A. micale

A. madytus, A. micale

M. malaitensis, $P$. citri

P. lilacinus

Tricentrus sp

Rachisphora sp.

$-$

$-$

$-$

A. micale

Coccid sp.

Scale insect $\mathrm{sp}$.

Icerya sp.

C. hesperidum [L. hesperidum]

$H$. bengalensis

Icerya sp.

O. tarandus $\mathrm{F}$.

L. painei

L. painei

Scale insect $\mathrm{sp}$.

Milviscutulus sp.

Sextius cf. 'kurandae'

H. phorbas

$-$

Milviscutulus $\mathrm{sp}$

A. micale

H. phorbas

Narathura araxes eupolis (Miskin)

\begin{tabular}{|c|c|c|}
\hline LYC & [29] & AUS \\
\hline- & [17] & AUS \\
\hline LYC & [29] & AUS \\
\hline- & [53] & SGP \\
\hline PSE & [40] & SLB \\
\hline PSE & [5] & MYS \\
\hline & & IVIYS \\
\hline ALE & [54] & IND \\
\hline _- & [16] & AUS \\
\hline- & [16] & AUS \\
\hline- & [16] & AUS \\
\hline - & [55] & MYS \\
\hline - & [16] & AUS \\
\hline - & [17] & AUS \\
\hline LYC & [29] & AUS \\
\hline $\mathrm{COC}$ & [52] & MYS \\
\hline- & [16] & AUS \\
\hline - & [17] & AUS \\
\hline $\mathrm{COC}+$ & [8] & AUS \\
\hline - & [35] & MYS \\
\hline- & [43] & MYS \\
\hline - & {$[6,7]$} & AUS \\
\hline MAR & [8] & AUS \\
\hline $\mathrm{COC}$ & [30] & IND \\
\hline TET & [30] & IND \\
\hline MAR & [30] & IND \\
\hline MEM & [30] & IND \\
\hline PSE & [40] & SLB \\
\hline PSE & [40] & SLB \\
\hline $\mathrm{COC}+$ & [45] & AUS \\
\hline- & [17] & AUS \\
\hline $\mathrm{COC}$ & [8] & AUS \\
\hline MEM & [8] & AUS \\
\hline LYC & [29] & AUS \\
\hline- & {$[16]$} & AUS \\
\hline- & [16] & AUS \\
\hline $\mathrm{COC}$ & [8] & AUS \\
\hline LYC & [29] & AUS \\
\hline LYC & [29] & AUS \\
\hline LYC & [29] & AUS \\
\hline LYC & [56] & AUS \\
\hline
\end{tabular}




\begin{tabular}{|c|c|c|}
\hline $\mathrm{Fam}^{2}$ & References & Ctry ${ }^{3}$ \\
\hline LYC & [29] & AUS \\
\hline LYC & [29] & AUS \\
\hline LYC & [29] & AUS \\
\hline- & [32] & AUS \\
\hline LYC & [29] & AUS \\
\hline LYC & [29] & AUS \\
\hline- & {$[6,7]$} & AUS \\
\hline- & {$[6,7]$} & AUS \\
\hline- & [32] & AUS \\
\hline LYC & [29] & AUS \\
\hline LYC & [29] & AUS \\
\hline- & {$[6,7]$} & AUS \\
\hline- & [13] & VNM \\
\hline - & {$[6,7]$} & AUS \\
\hline LYC & [29] & AUS \\
\hline- & {$[6,7]$} & AUS \\
\hline- & [32] & AUS \\
\hline - & {$[6,7]$} & AUS \\
\hline LYC & [29] & AUS \\
\hline LYC & [56] & AUS \\
\hline- & {$[6,7]$} & AUS \\
\hline PSE & [48] & PHL \\
\hline LYC & [28] & LKA \\
\hline- & [57] & MYS \\
\hline LYC & [29] & AUS \\
\hline LYC & [29] & AUS \\
\hline $\mathrm{COC}$ & [8] & AUS \\
\hline ALE & [54] & IND \\
\hline- & [58] & LKA \\
\hline- & [30] & IND \\
\hline- & [16] & AUS \\
\hline LYC & [29] & AUS \\
\hline- & {$[6,7]$} & AUS \\
\hline- & [59] & THA \\
\hline- & [59] & THA \\
\hline - & [59] & $\mathrm{TH} A$ \\
\hline $\mathrm{COC}+$ & [8] & AUS \\
\hline- & {$[6,7]$} & AUS \\
\hline LYC & [29] & $A U$ \\
\hline LYC & [29] & $\mathrm{AU}$ \\
\hline- & {$[6,7]$} & $A U$ \\
\hline
\end{tabular}

(F. Muell.) K.D. Hill \& L.A.S. Johnson

T. miskini

B50. Corymbia ptychocarpa (F. Muell.) K.D. Hill \& L.A.S. Johnson A. centaurus

A. centaurus

T. miskini

Eucalyptus confertiflora F. Muell.

Eucalyptus drepanophylla F. Muell. ex Benth

T. miskini

A53. Eucalyptus foelscheana F. Muell.

A54. Eucalyptus miniata A. Cunn. ex Schauer

A55. Eucalyptus papuana F. Muell. Eucalyptus sp.

A56. Eucalyptus tectifica F. Muell.

A57. Eucalyptus tereticornis Sm.

A58. Eucalyptus tetrodonta F. Muell.

Eucalyptus torelliana F. Muell.

A59. Lophostemon lactifluus (F. Muell.) Peter G. Wilson \& J.T. Waterh.

A60. Lophostemon suaveolens (Sol. ex Gaertn.) Peter G. Wilson

$$
\text { \& J.T. Waterh. [Tristania suaveolens] }
$$

A61. Melaleuca leucadendra (L.) L.

B52. Melaleuca quinquenervia (Cav.) S.T. Blake

A62. Melaleuca viridiflora Sol. ex Gaertn.

D7. Psidium guajava L.

B53. Ristantia pachysperma (Bailey) Peter G. Wilson \& J.T. Waterh B54. Syzygium cormiflorum B.P.M. Hyland

* C5. Syzygium cumini (L.) Skeels

* A63. Syzygium 'erythrocalyx' B. Hyland Syzygium sp.aff. erythrocalyx

A64. Syzygium eucalyptoides (F. Muell.) B. Hyland

C6. Syzygium jambos (L.) Alston

A65. Syzygium megacarpum (Craib) N.C. Rathakrishnan \& N.C. Nair

A66. Syzygium samarangense (Blume) Merr. \& L.M. Perry

A67. Syzygium sayeri B.P.M. Hyland

A68. Syzygium suborbiculare (Benth.) T.G. Hartley \& L.M. Perry

B55. Syzygium tierneyanum (Benth.) T.G. Hartley \& L.M. Perry

B56. Syzygium wilsoni (F. Muell.) B. Hyland

A69. Xanthostemon paradoxus F. Muell.

$-$

A. centaurus

T. miskini

T. miskini

$-$

A. centaurus

$N$. araxes eupolis

P. lilacinus

Z. chrysomallus

A. micale

A. micale

Coccus sp., Milviscutulus sp.

Rachisphora sp.

A. micale

Coccid sp.

A. micale

A. seltutus, $H$. phorbas

- 
33. Pandanaceae

$-$

Passifloraceae

A71. Adenia heterophylla (Blume) Koord.

35. Phyllantaceae

A72. Breynia stipitata Mull. Arg

Bridelia tomentosa Blume [Briedelia tomentosa] [EUPH]

B57. Glochidion ferdinandi (Mull. Arg.) F.M. Bailey [EUPH] Glochidion philippicum (Cav.) C.B.Rob. [EUPH]

36. Proteaceae

A73. Cardwellia sublimis F. Muell.

Macadamia integrifolia Maiden \& Betche Macadamia tetraphylla L.A.S. Johnson

A74. Persoonia falcata R. Br.

37. Putranjivaceae

A75. Drypetes lasiogyna (F. Muell.) Pax \& K. Hoffm.

38. Rhamnaceae

A76. Ziziphus oenoplia (L.) Mill.

39. Rhizophoraceae

$$
\text { Bruguiera sp. }
$$

B58. Ceriops tagal (Perr.) C.B. Robb

Ceriops sp.

C7. Rhizophora mucronata (Lam.)

40. Rubiaceae

A77. Aidia cochinchinensis Lour. [Randia cochinchinensis]

D8. Coffea excelsa A. Chevalier

D9. Coffea robusta L. Linden Coffea sp.

A78. Ixora klanderiana F. Muell. [Ixora klanderana] xora pavetta Andrews

A79. Morinda citrifolia L. [COMB]

A80. Timonius timon (Spreng.) Merr. Uncaria sp.

41. Rutaceae

B59. Citrus aurantiifolia

(Christm.) Swingle [Citrus acida]

A81. Citrus limon (L.) Burm. f.

A82. Citrus maxima (Burm.) Merr

A83. Citrus reticulata Blanco
A. lycaenoides

A micale

Austrotartessus sp.

Coccus sp.

$N$. berenice

$N$. berenice

Coccid sp.

H. phorbas

Coccid sp.

Coccus viridis Green

C. viridis

C. viridis

C. viridis [Lecanium viridis Green]

Rachisphora sp.

$-$

C. viridis

Coccid sp.

Mealybug sp.

Mealybug sp.

Scale insect $\mathrm{sp}$ $-\quad[6,7]$

AUS

- [17]

AUS

-
LYC

[17]

AUS

$[29]$
$[29]$
$[16]$

AUS

AUS

AUS

CIC [8] AUS

$\mathrm{COC}$ [8]

[29]

LYC [29]

AUS

AUS

AUS

$-[6,7] \quad$ AUS

[17]

AUS

- [17]

AUS

COC [52] MYS

LYC [29] AUS

COC [52]

- [9]

MYS

THA

COC

$\mathrm{COC}$

COC

$\mathrm{COC}$

ALE

$-$

$-$

$\mathrm{COC} \quad[43]$

[17]

$[17]$
$[61]$
$[61]$
$[60]$

[60]

[17]

[54]

[53]

[43]

$-$

COC

PSE

PSE

COC+

AUS

MYS

MYS

MYS

IND

MYS

AUS

IND
SGP

SGP

AUS

MYS

MYS

$\mathrm{CHN}$

CHN

$\mathrm{CHN}$

VNM

VNM

$\mathrm{CHN}$

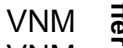


A85. Glycosmis trifoliata (Blume) Spreng.

A86. Micromelum minutum (G. Forst.) Seem. Murraya paniculata (L.) Jack Salicaceae

$$
\text { Flacourtia sp. [FLAC] }
$$

\section{Santalaceae}

A87. Exocarpos latifolius $\mathrm{R}$. Br.

\section{Sapindaceae}

* Alectryon coriaceus (Benth.) Radlk

Alectryon diversifolius (F. Muell.) S.T. Reynolds

Heterodendron diversifolium

Arytera divaricata F. Muell.

B60. Arytera pauciflora S.T. Reynolds $\begin{array}{ll}* & \text { Atalaya hemiglauca (F. Muell.) F. Muell. ex Benth. } \\ * & \text { Atalaya salicifolia (A.DC.) Blume }\end{array}$

Atalaya variifolia ( $F$. Muell.) Benth.

B61. Cupaniopsis anacardioides (A. Rich.) Radlk.

$$
\text { Cupaniopsis sp. }
$$

* A88. Litchi chinensis Sonn.

C8. Nephelium lappaceum L.

B62. Synima cordierii Radlk.

44. Sapotaceae

B63. Madhuca longifolia (L.) J.F. Macbr. [Bassia latifolia]

B64. Manilkara jaimiqui (C. Wright) Dubard subsp. emarginata (L.) Cronquist [Achras sapota]

A89. Pouteria sericea (Aiton) Baehni

\section{Smilacaceae}

B65. Smilax australis $\mathrm{R}$. $\mathrm{Br}$.

* Smilax cf. australis

46. Theaceae

B66. Camellia sinensis (L.) Kuntze
Mealybug sp.

Scale insect $\mathrm{sp}$

$-$

S. coffeae [L. hemisphaericum (S. hemisphaericum)]

N. berenice

N. berenice

N. berenice

A. seltutus

$N$. berenice

T. miskini

N. berenice

T. miskini

A. seltutus, A. micale, $H$. phorbas

A. lycaenoides, $N$. berenice

A. centaurus

A. lycaenoides

$-$

$-$

A. seltutus

C. viridis

C. viridis

$-$

H. phorbas

Coccus discrepans Green, C. hesperidum

Metaceronema japonica (Maskell)

[Eriochiton theae Green]

Fam $^{2}$ References Ctry
PSE $\mathrm{COC}+[62$

[30]

$[63$

[5]

$-$

[17]

COC

[51]

[17]

VNM

VNM
VNM


plant species with which existing perennial cropping systems could be enriched.

\section{Materials and Methods}

A survey of the literature was carried out to identify host plants and trophobionts recorded worldwide for 0 . smaragdina and $O$. longinoda. The literature survey was largely conducted on the $C A B$ Direct database that included international archives dating back to 1900. The search terms 'Oecophylla longinoda' and 'Oecophylla smaragdina' were used to obtain records for the two species. For the purpose of this survey, 'host plants' were those that the ant was reported to nest in, while 'possible host plants' had no confirmation of nesting. Where the abstract alluded to a possible host plant, the original article was reviewed, and where more than one reference was available for a host plant, the earliest mention was recorded, along with associated trophobionts (if any) and country of occurrence.

The plant names were checked against other standardized databases using the GRIN (Online) Taxonomic Nomenclature Checker (TNC) [18]. At the date of accession (30 September 2006), the database contained over 18000 generic and 65000 specific or infraspecific records of vascular plants and included all currently accepted generic names (over 14000). As representation of species in this database was incomplete, especially for non-agricultural plants, some of the plant names were checked against other sources [19-23]. The TNC highlighted species that did not match those in the database and provided up to five possible alternatives, based on which possible spelling mistakes in the original article could be corrected if a close match was found with a matching distributional range and family. Relevant articles cited by the ones found in the database search also provided additional host plant records. Scale insect trophobiont names were checked against the ScaleNet [24] database for scale insects of the world.

\section{Statistical Analyses}

Chi-square analyses of data of the host plants and associated trophobionts of $O$. longinoda and $O$. smaragdina were carried out using the statistical software Minitab $14{ }^{\circledR}$ [25]. The two ant species were compared in the distribution of host plant species by trophobiont taxon.

\section{Results}

The CAB Direct database search for 'Oecophylla longinoda' and 'Oecophylla smaragdina' returned 99 and 228 records, respectively. The literature survey showed that 0 . smaragdina was recorded on 175 plant species in 46 families, with 28 associated trophobiont species in 7 families (Table 1), whereas 0 . longinoda was recorded on 66 plant 
Table 2 Records of Oecophylla longinoda host plants and associated trophobionts from a survey of the literature (19002006). Records are from Way [4] in Zanzibar unless indicated otherwise in curly brackets \{\} . Currently accepted species and family names are used followed by names given in the original article in square brackets [ ], where different

\author{
Host plant species ${ }^{1}$ \\ 1. Anacardiaceae \\ F1. Anacardium occidentale L. \\ D1. Mangifera indica L. \\ 2. Annonaceae \\ D2. Annona muricata L. \\ F2. Annona senegalensis Pers. \\ [Annona chrysophylla] \\ F3. Canangium odoratum (Lam.) Baill. ex King \\ 3. Apocynaceae \\ F4. Rauvolfia mombasiana Stapf [Rauwolfia \\ mombasiana] \\ F5. Schizozygia coffaeoides Baill. \\ [Schizozygia coffeoides] \\ 4. Arecaceae \\ D3. Areca catechu L. [PALM] \\ D4. Cocos nucifera L. [PALM]
}

\section{Asteraceae}

Ageratum sp. [Compositae]

5. Bignoniaceae

F6. Millingtonia hortensis L. f.

6. Boraginaceae

E1. Cordia aurantiaca Baker [Cordia aurentiaca] \{[67], Cameroon\}

7. Burseraceae

F7. Canarium commune L.

8. Colchicaceae

F8. Gloriosa simplex Linn. [Liliaceae]

9. Combretaceae

C1. Terminalia catappa L.

10. Cucurbitaceae

F9. Momordica foetida Schumach.

11. Euphorbiaceae

E2. Alchornea laxiflora (Benth.) Pax \& K. Hoffm. $\{[69]$, Kenya\}

Codiaeum sp.

12. Fabaceae

F10. Acacia glauca (L.) Moench [Leucaena glauca] [LEGU]

E3. Afzelia quanzensis Welw. [CAES] \{[68], Kenya\}

F11. Bauhinia thonningii Schumach. [LEGU]

\section{Cassia sp. [LEGU]}

D5. Delonix regia (Bojer ex Hook.) Raf. [LEGU]

F12. Gliricidia sepium (Jacq.) Kunth ex Walp. [LEGU]
Associated trophobiont

$\mathrm{Fam}^{2}$

Coccus sp. nr. hesperidum L., Parasaissetia nigra (Neitn.) [Saissetia. nigra (Nietn.)], Saissetia zanzibarensis Williams

Pseudococcus sp.

Coccus hesperidum L., Saissetia sp. nr. nigra (Nietn.), S. zanzibarensis

Pseudococcus sp., Rastrococcus iceryoides (Green) [Phenacoccus iceryoides Green]

$\begin{array}{ll}\text { Parasaissetia sp. nr. nigra [Saissetia sp. nr. nigra] } & \text { COC } \\ \text { Parastictococcus anonae (Green and Laing) } & \text { STI }\end{array}$ Parastictococcus anonae (Green and Laing)

[Stictococcus anonae Green and Laing]

Isthmia sp.

$P$. nigra [Saissetia sp. ?nigra]

$P$. anonae [S. anonae]

Coccus viridis Green, Saissetia sp. nr. coffeae (Wlk.), Udinia sp. nr. catori (Green) [Saissetia sp. nr. catori (Green)]

Pseudococcus sp.

Membracid sp.

$R$. iceryoides $[P$. iceryoides]

$\mathrm{COC}$

S. zanzibarensis

Cerataphis lataniae Boisduval

C. hesperidum, P. nigra [S. nigra], S. zanzibarensis

Planococcus sp., Pseudococcus cryptus Hempel [Pseudococcus citriculus Green]

Parasaissetia sp. nr. nigra [Saissetia sp. nr. nigra] Pseudococcus sp.

PSE

$\mathrm{COC}$

PSE

TET

$\mathrm{COC}$ STI

COC

PSE

MEM PSE

COC

$\mathrm{APH}$

COC

PSE

COC

PSE

Udinia sp. nr. catori [Saissetia sp. nr. catori]

COC

Membracid sp.

MEM

S. zanzibarensis

$\mathrm{COC}$

S. zanzibarensis

$\mathrm{COC}$

Membracid sp.

MEM

Saissetia sp.

COC

Saissetia sp. nr. coffeae

COC

Pseudococcus sp.

PSE

C. hesperidum

COC

C. hesperidum

Membracid sp.

S. zanzibarensis

Membracid sp.

S. zanzibarensis

$P$. nigra [S. nigra]

$R$. iceryoides $[P$. iceryoides]

S. zanzibarensis
$-$

COC

MEM

COC

MEM

$\mathrm{COC}$

COC

PSE

COC 
Table 2 (Continued)

\begin{tabular}{cll}
\hline Host plant species $^{1}$ & Associated trophobiont & Fam $^{2}$ \\
\hline E4. Julbernardia magnistipulata [CAES] ([68], Kenya) & - & - \\
$\begin{array}{c}\text { E5. Pithecellobium dulce (Roxb) Benth. [Pithecollobium } \\
\text { dulce] [LEGU] }\end{array}$ & Membracid sp. & MEM \\
F13. Tephrosia vogelii Hook. f. [LEGU] & C. hesperidum & COC
\end{tabular}

13. Hypericaceae

F14. Harungana madagascariensis Lam. ex Poir.

E6. Vismia orientalis Engl. \{[68], Kenya\}

14. Icacinaceae

E7. Apodytes dimidiata E. Mey. ex Bernh. \{[68], Kenya\}

15. Lamiaceae

F15. Clerodendrum glabrum E. May [Clerodendron glabrum, VERB]

D6. Tectona grandis L. f. [VERB]

F16. Vitex doniana Sweet [VERB]

16. Lauraceae

F17. Cassytha filiformis L.

D7. Persea americana Mill.

17. Lecythidaceae

F18. Barringtonia racemosa (L.) Spreng.

18. Loranthaceae

E8. Loranthus sansibarensis Engl.

19. Lythraceae

F19. Sonneratia alba Sm. (Sonneratia caseolaris) [SONN]

20. Malvaceae

F20. Adansonia digitata L. [BOMB]

F21. Durio zibethinus L. [BOMB]

F22. Grewia glandulosa Vahl [TILI]

E9. Malvaviscus grandiflorus H.B. \& K

D8. Theobroma cacao L. [STER]

$$
\text { T. cacao L. ([69], Cote D'Ivoire) }
$$

Meliaceae

Turraea sp. \{[68], Kenya\}

21. Moraceae
D9. Artocarpus heterophyllus Lam.
Ficus spp.

22. Myrtaceae

F23. Eucalyptus camaldulensis Dehnh.

D10. Psidium guajava L.

F24. Syzygium aromaticum (L.) Merr. \& L. M. Perry

[Jambosa caryophyllus (Eugenia aromatica)]

D11. Syzygium cumini (L.) Skeels (Eugenia jambolana)
C. viridis, Parasaissetia sp. nr. nigra [Saissetia sp. COC nr. nigra], S. zanzibarensis

Pseudococcus sp.

Xiphistes sp.

PSE

MEM

$-$

$-$

S. zanzibarensis $\quad \mathrm{COC}$

C. hesperidum COC

Membracid sp.

C. hesperidum, Saissetia sp. nr. coffeae COC

Saissetia sp. nr. oleae (Olivier) [Saissetia sp. nr. COC oleae (Bern.)]

C. hesperidum, S. zanzibarensis COC

Parasaissetia sp. nr. nigra [Saissetia sp. nr. nigra], COC Parthenolecanium sp. nr. persicae (Fabricius) [Coccus sp. nr. elongatus (Sign.)]

Membracid sp.

MEM

Saissetia sp. $\quad$ COC

P. nigra [S. nigra], Saissetia sp. nr. oleae (Olivier) COC [Saissetia sp. nr. oleae (Bern.)]

Margarodid sp. MAR

Parthenolecanium sp. nr. persicae COC

[Coccus sp. nr. elongatus], S. zanzibarensis,

Udinia sp. nr. catori [Saissetia sp. nr. catori]

Parthenolecanium sp. nr. persicae

[Coccus sp. nr. elongatus], Udinia sp. nr. catori

[Saissetia sp. nr. catori]

Margarodid $\mathrm{sp}$.

$R$. iceryoides [P. iceryoides]

Coccid sp.

Pseudococcus sp., R. iceryoides [P. iceryoides (Phenacoccus iceryoides Green)]

Toxoptera sp. ? aurantii (Boy)

Stictococcid sp.

$\mathrm{COC}$

MAR

PSE

COC

PSE

$\mathrm{APH}$

STI

C. hesperidum

Margarodid sp.

$P$. nigra [S. nigra], S. zanzibarensis

$R$. iceryoides $[P$. iceryoides]

Coccus sp. ?hesperidum, C. viridis,

S. zanzibarensis

C. viridis, S. zanzibarensis

Pseudococcus sp.

Coccus sp. nr. hesperidum, C. viridis,

Eulecanium sp., Parasaissetia sp. nr. nigra

[Saissetia sp. nr. nigra], Saissetia sp. nr. coffeae,

S. zanzibarensis

Coccus sp. nr. viridis, S. zanzibarensis

Membracid sp.

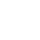

.

.

\section{.}

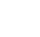


Table 2 (Continued)

\begin{tabular}{|c|c|c|}
\hline Host plant species ${ }^{1}$ & Associated trophobiont & $\mathrm{Fam}^{2}$ \\
\hline \multirow[t]{2}{*}{$\begin{array}{l}\text { D12. Syzygium jambos (L.) Alston [Jambosa jambos } \\
\text { (Eugenia jambos)] }\end{array}$} & $\begin{array}{l}\text { Coccus sp. nr. hesperidum, Eucalymnatus } \\
\text { tessellatus (Sign.), S. zanzibarensis }\end{array}$ & $\mathrm{COC}$ \\
\hline & Membracid sp. & MEM \\
\hline $\begin{array}{l}\text { E10. Syzygium malaccense (L.) Merr. \& L.M. Perry } \\
\text { [Jambosa malaccensis (Eugenia malaccensis)] }\end{array}$ & Membracid sp. & MEM \\
\hline \multicolumn{3}{|l|}{ 23. Olacaceae } \\
\hline E11. Olax dissitiflora Oliv. $\{[68]$, Kenya $\}$ & - & - \\
\hline \multicolumn{3}{|l|}{ 24. Oleaceae } \\
\hline F25. Jasminum fluminense Vell. & S. zanzibarensis & $\mathrm{COC}$ \\
\hline \multicolumn{3}{|l|}{ 25. Oxalidaceae } \\
\hline F26. Averrhoa bilimbi L. & $\begin{array}{l}\text { Pseudococcid sp. } \\
\text { S. zanzibarensis }\end{array}$ & $\begin{array}{l}\text { PSE } \\
\text { COC }\end{array}$ \\
\hline F27. Averrhoa carambola L. & S. zanzibarensis & $\mathrm{COC}$ \\
\hline \multicolumn{3}{|l|}{ 26. Passifloraceae } \\
\hline F28. Passiflora quadrangularis L. & $P$. nigra $[S$. nigra] & $\mathrm{COC}$ \\
\hline \multicolumn{3}{|l|}{ 27. Phyllanthaceae } \\
\hline F29. Bridelia micrantha (Hochst.) Baill. [EUPH] & $\begin{array}{l}\text { Membracid sp. } \\
P . \text { nigra }[S . \text { nigra] }\end{array}$ & $\begin{array}{l}\text { MEM } \\
\text { COC }\end{array}$ \\
\hline E12. Uapaca alluminata [CAES] \{[67], Cameroon $\}$ & - & - \\
\hline \multicolumn{3}{|l|}{ 28. Rhamnaceae } \\
\hline E13. Ziziphus mauritiana Lam. & Pseudococcid sp. & PSE \\
\hline \multicolumn{3}{|l|}{ 29. Rhizophoraceae } \\
\hline Ceriops sp. & P. nigra [S. nigra], S. zanzibarensis & $\mathrm{COC}$ \\
\hline D13. Rhizophora mucronata (Lam.) & S. zanzibarensis & $\mathrm{COC}$ \\
\hline \multicolumn{3}{|l|}{ 30. Rosaceae } \\
\hline E14. Eriobotrya japonica (Thunb.) Lindl. & Coccid sp. & $\mathrm{COC}$ \\
\hline \multicolumn{3}{|l|}{ 31. Rubiaceae } \\
\hline \multirow[t]{2}{*}{ F30. Canthium zanzibaricum Klotzsch } & $\begin{array}{l}\text { C. hesperidum, Parasaissetia sp. nr. nigra } \\
\text { [Saissetia sp. nr. nigra], S. zanzibarensis }\end{array}$ & $\mathrm{COC}$ \\
\hline & Pseudococcus sp. & PSE \\
\hline F31. Chassalia umbraticola Vatke & C. viridis & $\mathrm{COC}$ \\
\hline D14. Coffea excelsa A. Chevalier & C. viridis, S. zanzibarensis & $\mathrm{COC}$ \\
\hline F32. Coffea liberica Bull. ex K. Shum. & C. viridis, P. nigra [S. nigra], S. zanzibarensis & $\mathrm{COC}$ \\
\hline D15. Coffea robusta L. Linden & C. viridis, S. zanzibarensis & $\mathrm{COC}$ \\
\hline Polysphaeria sp. & C. viridis & $\mathrm{COC}$ \\
\hline \multicolumn{3}{|l|}{ 32. Rutaceae } \\
\hline Citrus, five spp. & C. lantaniae & $\mathrm{APH}$ \\
\hline & $\begin{array}{l}\text { C. hesperidum, C. viridis, S. zanzibarensis } \\
\text { Icerya seychellarum (Westw.) }\end{array}$ & $\begin{array}{l}\text { COC } \\
\text { MAR }\end{array}$ \\
\hline & $\begin{array}{l}\text { Planococcus citri, P. cryptus [P. citriculus], } \\
\text { Pseudococcus } \mathrm{sp} \text {. }\end{array}$ & PSE \\
\hline E15. Murraya paniculata (L.) Jack & Coccid sp. & $\mathrm{COC}$ \\
\hline \multicolumn{3}{|l|}{ 33. Sapindaceae } \\
\hline \multirow[t]{2}{*}{ D16. Nephelium lappaceum L. } & $\begin{array}{l}\text { C. hesperidum L., Udinia sp. nr. catori [Saissetia sp. } \\
\text { nr. catori] }\end{array}$ & $\mathrm{COC}$ \\
\hline & P. anonae [S. anonae] & STI \\
\hline E16. Paullinia pinnata L. & Pseudococcid sp. & PSE \\
\hline 34. Sapotaceae & & \\
\hline F33. Achras zapotilla (Jacq.) Nutt. & C. viridis, Saissetia sp. nr. coffeae, S. zanzibarensis & $\mathrm{COC}$ \\
\hline
\end{tabular}

${ }^{1}$ Groups: C and D, Oecophylla spp. host plants without and with trophobionts identified to species, respectively; E and F, O. longinoda host plants without and with trophobionts Oidentified to species, respectively (Figure 1). Plant family name abbreviations after Kiger and Reveal [26]. Numbering of host plant families only for plants identified to species in each family.

${ }^{2}$ Families: APH, Aphididae; COC, Coccidae; MAR, Margarodidae; MEM, Membracidae; PSE, Pseudococcidae; STI, Stictococcidae; TET, Tettigometridae.

species in 34 families with 17 associated trophobiont species in 6 families (Table 2). In addition, there were 73 species (22 families) of possible host plants (i.e. nesting not confirmed) for $O$. smaragdina (Table 1$)$. A number of these possible host plants had been reported as host plants also, but the distinction was maintained because their associated trophobiont species were different. Trophobiont association may influence a plant species' suitability as a host plant for the weaver ant.

The large number of host plant records for 0 . smaragdina compared with $O$. longinoda was due in part to an extensive checklist of host plants of lepidopteran species 
Table 3 Percentage of host plant species recorded in the literature for trophobionts tended by Oecophylla smaragdina and Oecophylla longinoda, and the distribution of trophobiont species by taxon. Number of species in parentheses

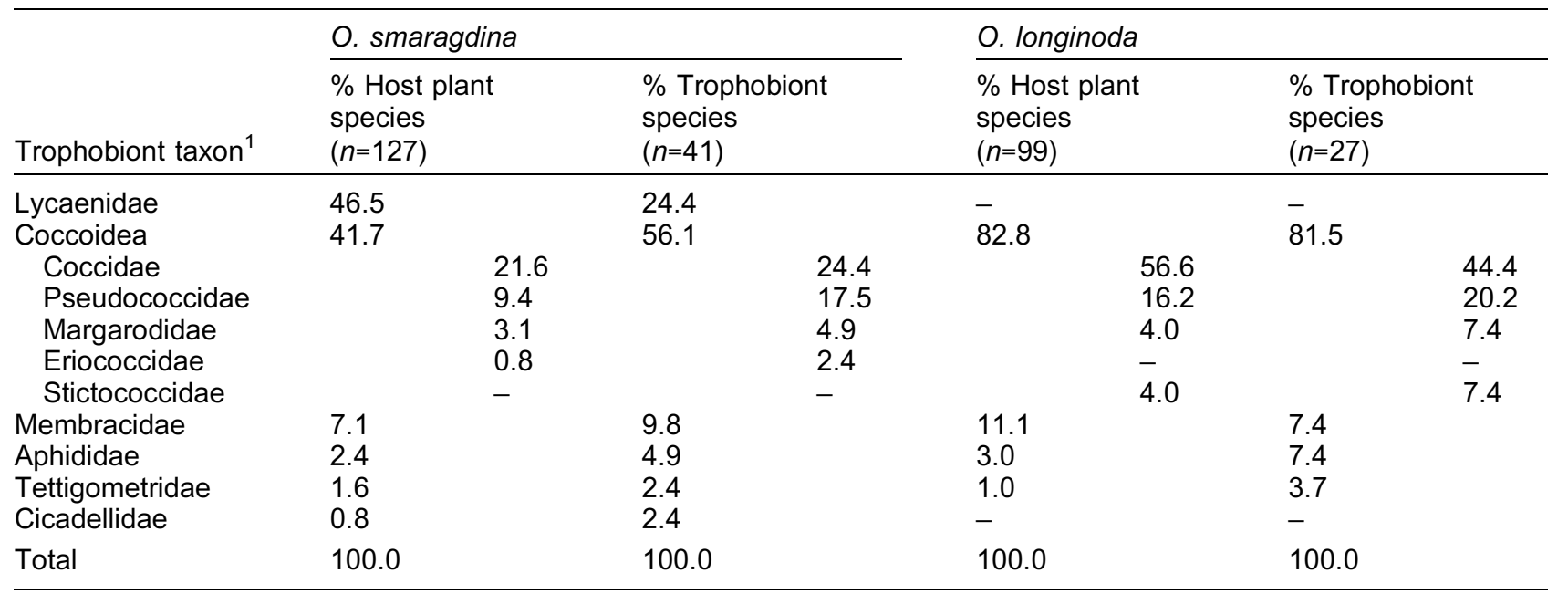

${ }^{1}$ Scale insect families further grouped under the superfamily Coccoidea.

in the family Lycaenidae [29]. Lycaenid larvae secrete nutritious rewards for ants [70] and several lycaenid species are obligately tended by 0 . smaragdina [29], while no lycaenids have been reported for $O$. longinoda. Lycaenid host plants consisted of as much as $46.5 \%$ of the host plant species recorded for 0 . smaragdina, but contributed only $24.4 \%$ to the total number of trophobiont species reported for the ant (Table 3). Excluding the family Lycaenidae, there was no significant difference between the two ant species, in the number of trophobiont species reported for Coccidae, Pseudococcidae, other scale insect families, and other trophobiont families $\left(\chi^{2}=1.000\right.$; d.f. $=1,3 ; P=0.801)$.

Figure 1 summarizes the number of (confirmed) host plant species and families for the two ant species, and the number of trophobiont species associated with the ant species. Trophobionts were listed on less than half of the host plants recorded for $O$. smaragdina (various references) but on most host plants recorded for $O$. longinoda, many of which were listed in Way [4]. There were 17 plant species on which both ant species were reported nesting (Table 4). Eleven of the plant species reported trophobiont associations but only Coffea excelsa A. Chevalier (Rubiaceae) and Coffea robusta $\mathrm{L}$. Linden shared a common trophobiont, Coccus viridis Green (Coccidae) tended by both ant species. $C$. viridis is a scale insect found on many plant species around the world, including 7 [24] of the 17 plant species hosting both Oecophylla spp. This scale insect was reported as a coffee pest in Sri Lanka, particularly on disease-weakened plants. $C$. viridis tended by $O$. smaragdina in Malaya did not cause apparent damage to healthy coffee plants but preventive measures were taken to destroy the ants and scale insects because the ants bit coffee pickers and were perceived to spread scales [61].

Six other trophobiont species were reported for both Oecophylla spp. but associated with different host plants:

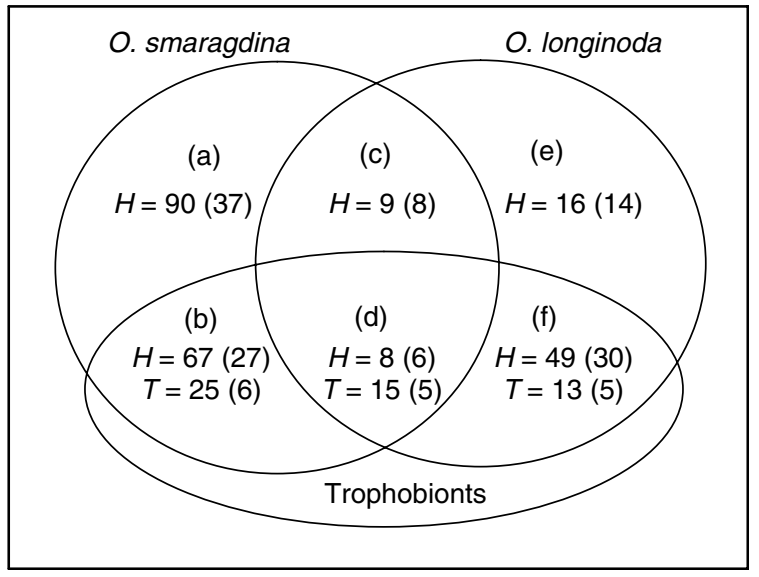

Figure 1 Number of host plant species, plant families and trophobiont species reported in the literature for Oecophylla smaragdina and Oecophylla longinoda. $H$, number of host plant species; $T$, number of trophobiont species for the host plant species. The number of families is stated in parentheses. There were 17 host plant species common to both Oecophylla spp. (subsets (c) and (d)). For these 17 host plant species, trophobionts were recorded on 8 and 16 plant species hosting $O$. smaragdina and $O$. longinoda, respectively (indicated by group $\mathrm{D}$ in Tables 1 and 2, respectively)

Planococcus citri (Risso) (Pseudococcidae), Coccus hesperidum L., Saissetia coffeae (Walker) and Parasaissetia nigra (Neitner) (Coccidae), Toxoptera aurantii (Boyer de Fonscolombe) and Cerataphis lataniae Boisduval (Aphididae). Except for $C$. lataniae, an ornamental pest that has a relatively small host range [71], these trophobionts are polyphagous and widely distributed [24]. $P$. citri damages a wide range of crops, some by vectoring diseases, while C. hesperidum, S. coffeae, P. nigra and T. aurantii are pests of citrus, ornamentals, litchi, and coffee and citrus, respectively. The aphids potentially vector plant viruses as well. 
Table 4 Host plant species common to Oecophylla smaragdina and Oecophylla longinoda reported in a CAB database literature search (1900-2007)

\begin{tabular}{|c|c|c|}
\hline Host plant species & Family & Common name \\
\hline Annona muricata & Annonaceae & Soursop, anona \\
\hline Areca catechu & Arecaceae & Areca \\
\hline $\begin{array}{l}\text { Artocarpus } \\
\text { heterophyllus }\end{array}$ & Moraceae & Jackfruit \\
\hline Cocos nucifera & Arecaceae & Coconut \\
\hline Coffea excelsa & Rubiaceae & Coffee \\
\hline Coffea robusta & Rubiaceae & Coffee \\
\hline Delonix regia & Fabaceae & Flame tree \\
\hline Mangifera indica & Anacardiaceae & Mango \\
\hline Nephelium lappaceum & Sapindaceae & Rambutan \\
\hline Persea americana & Lauraceae & Avocado \\
\hline Psidium guajava & Myrtaceae & Guava \\
\hline Rhizophora mucronata & Rhizophoraceae & Mangrove \\
\hline Syzygium cumini & Myrtaceae & $\begin{array}{l}\text { Java plum, } \\
\text { jamun }\end{array}$ \\
\hline Syzygium jambos & Myrtaceae & Rose apple \\
\hline Tectona grandis & Lamiaceae & Teak \\
\hline Terminalia catappa & Combretaceae & $\begin{array}{l}\text { Tropical } \\
\text { almond }\end{array}$ \\
\hline Theobroma cacao & Malvaceae & Cocoa \\
\hline
\end{tabular}

O. smaragdina was a nuisance pest in tea plantations and jackfruit orchards in India $[66,72]$ because the ants bit workers. As in the case of $C$. viridis on coffee, hemipteran insects tended by weaver ants normally did not noticeably damage clove, tea, rubber and jackfruit [47, 66, 72, 73]. However, large populations on very young coffee plants caused some yellowing [4]. A large $O$. longinoda colony confined to a single tree was also observed to have damaging scale population levels [74]. O. longinoda was shown to distribute scale insects on available plants such that the plants were not affected by scale insect feeding. Further, culling of excessive scale insects by $O$. longinoda maintains trophobiont populations at a level that would meet colony honeydew needs [73]. Nevertheless, control measures were often advocated to prevent the homopterans from being spread by their custodians [47, 66, 72, 73]. In addition to being a nuisance pest, 0 . smaragdina was found to deter insect pollinators of Nephelium lappaceum L. (Sapindaceae) but no apparent reduction in fruit yield was observed [65]. The ant also preys on honeybees (Apis mellifera L., Hymenoptera) [4].

\section{Discussion}

The number of host plant species reported for 0 . smaragdina was more than double that for 0 . longinoda, suggesting a greater research interest toward the former or a wider geographic distribution with a more diverse plant community. Recent contributions to the $O$. smaragdina host plant species and trophobiont records [6-8, 29] comprized over half the records and reflect the current research interest toward this ant species. In contrast, the majority of host plant species for 0 . longinoda were reported in 1954 and was likely biased towards plants with trophobionts [4]. The interest displayed for 0 . smaragdina has largely been in the context of its application as a biological control agent where the ant is a valued tool in Asian and Australian IPM systems [75].

The present study also revealed that the 17 host plant species common to both ant species were largely fruit or cash crop species cultivated in the tropics around the world. Furthermore, about half the host plant species reported for either ant species are economically important or of value to human society. Thus, there is a large potential pool of value-added host plant species from which candidates that favour either ant species may be chosen. The applicability of the ants to protecting these plants depends on how the plants are utilized where grown. For example, in the Can Tho province, kapok (Ceiba pentandra) trees are either lopped for leaves used to produce incense sticks or left as is to produce kapok [76]. Weaver ants are unlikely to nest in regularly lopped kapok trees. Therefore, when selecting trees to promote certain ecosystem functions, local practices need to be considered [77].

It is apparent that the trophobiont species of Oecophylla spp. are not well documented yet. The two ant species appeared to associate with several trophobiont taxa, particularly those from the family Membracidae and superfamily Coccoidea. The trophobiont species common to both Oecophylla spp. were generally highly polyphagous and occur on a large number of plant species that host these ants [24] but have not yet been recorded as such. The pest risk of trophobionts found on these plant species may not be significant on healthy mature trees as indicated by observations on tea, coffee, rubber, jackfruit and clove noted earlier. The scarce mention and limited abundance of $O$. smaragdina-tended trophobionts on citrus crops where the ant has been used $[3,13]$ and the widespread use of this ant suggests that trophobionts do not pose a notable pest risk for some crops. The ants may keep their trophobiont population levels in check. It was also suggested that the $O$. longinoda preferentially tended non-pest Stictococcids rather than the pest-prone Pseudococcids [4]. However, where trophobionts may cause direct damage, e.g. when concentrated on developing fruit of cocoa [4] and mango [78], cost-benefit analyses comparing ant-treated with conventionally managed crops are needed. For example, mealybug damage was found to be greater for weaver ant-occupied mango trees compared with insecticide-sprayed trees, but overall control of pests was equally good [78].

The practical application of the weaver ants to crop protection is also dependent on human perception and acceptance. While trophobionts of the weaver ant, e.g. scales in mango, are perceived as pests or insignificant at best by western scientists [75], fruit pickers associate the presence of scales on mango as an indication of quality 
fruit in Benin (Paul van Mele, personal communication). In Vietnam, which has a long-standing tradition of weaver ant application to crop protection, locals regard the ant favourably and have devised methods, e.g. harvesters apply ash to arms and hands, for harvesting fruit that minimize ant bites [10]. In Australia, mango pickers immediately immerse harvested fruit in buckets of water to remove ants and spray trees with water prior to harvesting to reduce ant activity [78]. In Malaysia however, public opinion appears divided as to the beneficial versus pest status of the weaver ant (G. T. Lim, personal observation). While acknowledging the protective effects of the ants on fruit trees, many people consider the ant a pest because it bites and makes harvesting fruit difficult.

\section{Acknowledgements}

We thank Paul van Mele and the anonymous reviewers for commenting on earlier drafts. This paper is a partial result of a Virginia Tech PhD dissertation by the first author, who was jointly sponsored by the Fulbright programme, the Malaysian-American Commission for Educational Exchange, Virginia Tech and FRIM.

\section{References}

1. Hölldobler B. Territorial behavior in the green tree ant (Oecophylla smaragdina). Biotropica 1983;15:241-50.

2. Ledoux A. Rechercher sur la biologie de la fourmi fileuse (Oecophylla longinoda Latr.). Annales des sciences naturelles. Zoologie et biologie animale 1950;12:333-461.

3. Huang HT, Yang P. The ancient cultured citrus ant. BioScience 1987;37:655-71.

4. Way MJ. Studies of the life history and ecology of the ant Oecophylla longinoda Latreille. Bulletin of Entomological Research 1954;45:93-112.

5. Way MJ, Khoo KC. Colony dispersion and nesting habits of the ants, Dolichoderus thoracicus and Oecophylla smaragdina (Hymenoptera: Formicidae), in relation to their success as biological control agents on cocoa. Bulletin of Entomological Research 1991;81:341-50.

6. Peng RK, Christian K, Gibb K. Distribution of the green ant, Oecophylla smaragdina (F.) (Hymenoptera: Formicidae), in relation to native vegetation and the insect pests in cashew plantations in Australia. International Journal of Pest Management 1997;43:203-11.

7. Peng RK, Christian K, Gibb K. Control threshold analysis for the tea mosquito bug, Helopeltis pernicialis (Hemiptera: Miridae) and preliminary results concerning the efficiency of control by the green ant, Oecophylla smaragdina (Hymenoptera: Formicidae) in northern Australia. International Journal of Pest Management 1997;43:233-7.

8. Blüthgen N, Fiedler K. Interactions between weaver ants Oecophylla smaragdina, homopterans, trees and lianas in an Australian rain forest canopy. Journal of Animal Ecology 2002;71:793-801.
9. Offenberg J, Havanon S, Aksornkoae S, Maclntosh DJ, Nielsen MG. Observations on the ecology of weaver ants (Oecophylla smaragdina Fabricius) in a Thai mangrove ecosystem and their effect on herbivory of Rhizophora mucronata Lam. Biotropica 2004;36:344-51.

10. Van Mele P, Cuc NTT. Ants as Friends: Improving Your Tree Crops with Weaver Ants. CABI Bioscience; 2003. p. 67. ISBN 958-97218-2-6.

11. Peng RK, Christian K, Gibb K. Implementing Ant Technology in Commercial Cashew Plantations and Continuation of Transplanted Green Ant Colony Monitoring. RIRDC, Australia; 2004. Available from: URL: http://www.rirdc.gov.au/ reports/NPP/04-088.pdf (accessed 6 March 2007).

12. Peng RK, Christian K, Gibb K. The effect of colony isolation of the predacious ant, Oecophylla smaragdina (F.) (Hymenoptera: Formicidae), on protection of cashew plantations from insect pests. International Journal of Pest Management 1999;45:189-94.

13. Van Mele P, Cuc NTT. Evolution and status of Oecophylla smaragdina as a pest control agent in citrus in the Mekong Delta, Vietnam. International Journal of Pest Management 2000;46:295-301.

14. Lim GT, Kirton LG. A preliminary study on the prospects for biological control of the mahogany shoot borer, Hypsipyla robusta (Lepidoptera: Pyralidae), by ants (Hymenoptera: Formicidae). In: Proceedings of the International Conference on Forestry and Forest Products Research. FRIM, Malaysia; 2003. p. $240-4$.

15. Van Mele P, Vaysierres JF, Tellingen EV, Vrolijks J. Effects of an African weaver ant in controlling mango fruit flies (Diptera: Tephritidae) in Benin. Journal of Economic Entomology 2007;100:695-701.

16. Blüthgen N, Stork NE, Fiedler K. Bottom-up control and co-occurrence in complex communities: honeydew and nectar determine a rainforest ant mosaic. Oikos 2004; 106:344-58.

17. Begg RJ. The effects of cyclone 'Tracy' on the ant Oecophylla smaragdina. Journal of the Australian Entomological Society 1977;16:289-95.

18. USDA-ARS. Taxonomic Nomenclature Checker - GRIN [online]. 2006. Available from: URL: http://pgrdoc.ipgri.cgiar. org/taxcheck/grin/index.html (accessed 30 September 2006).

19. RBG. Flora Zambesiaca. Board of Trustees of the Royal Botanic Gardens, Kew; 2004. Availabe from: URL: http://www.rbgkew.org.uk/floras/fz/intro.html (accessed 30 September 2006).

20. The International Plant Names Index (IPNI) 2004. Available from: URL: http://www.ipni.org (accessed 29 October 2006).

21. DOL. 2005. PlantSystematics.org. DiversityofLife.org, http:// 132.236.163.181/index.html (accessed 30 September 2006).

22. Western Australian Herbarium. FloraBase (ver 2.1.3) - The Western Australian Flora. Department of Environment and Conservation; 2006. Available from: URL: http:// florabase.calm.wa.gov.au/ (accessed 30 September 2006).

23. APNI. The Australian Plant Name Index. ANBG; 2006. Available from: URL: http://www.anbg.gov.au/apni/apni.html (accessed 30 September 2006).

24. Ben-Dov Y, Miller DR, Gibson GAP. ScaleNet: Query Index. 2005. Available from: URL: http://www.sel.barc.usda.gov/ scalenet/query.htm (accessed 1 November 2006). 
25. MINITAB. MINITAB Statistical Software Release 14.20; 2007.

26. Kiger RW, Reveal JL. A Comprehensive Scheme for Standardized Abbreviation of Usable Plant-family Names and Type-based Suprafamilial Names. 2006. Available from: URL: http://www.chemlife.umd.edu/emeritus/reveal/pbio/fam/ famabbr.html (accessed 5 May 2007)

27. Corbett GH. Division of Entomology. Annual report for the year 1936. 26, Department of Agriculture, South Seas and Federated Malay States, General Series/Department of Agriculture, Straits Settlement; 1937.

28. van der Poorten M, van der Poorten N. Butterflies and Dragonflies of Sri Lanka. 2006. Available from: URL: http://www.srilankaninsects.net/Butterflies/Lycaenidae/ Redspot/RedSpot.htm (accessed 30 September 2006).

29. Braby MF. 2000. Family Lycaenidae. In: Butterflies of Australia. CSIRO Publishing, Canberra; 2000. p. 617-856.

30. Dutt GR. Life histories of Indian insects, IV (Hymenoptera): Oecophylla smaragdina F. Memoirs of the Department of Agriculture in India (Entomology); 1912. p. 254-60.

31. Van Mele P, Cuc NTT, Van Huis A. Farmers' knowledge, perceptions and practices in mango pest management in the Mekong Delta, Vietnam. International Journal of Pest Management 2001;47:7-16.

32. Lokkers C. The distribution of the weaver ant, Oecophylla smaragdina (Fabricius) (Hymenoptera: Formicidae) in Northern Australia. Australian Journal of Zoology 1986; 34:683-7.

33. Van Mele P, Cuc NTT. Predatory ants in orchards in the Mekong Delta of Vietnam. In: Loke WH, editor. Symposium on Biological Control in the Tropics. CABI, Serdang, Malaysia; 1999. p. $118-22$.

34. Stapley JH. Using the predatory ant, Oecophylla smaragdina, to control insect pests of coconuts and cocoa. Information Circular - South Pacific Commission no. 85. 1980.

35. Saarinen EV. Differences in worker caste behaviour of Oecophylla smaragdina (Hymenoptera: Formicidae) in response to larvae of Anthene emolus (Lepidoptera: Lycaenidae). Biological Journal of the Linnean Society 2006;88:391-5.

36. More PS, Desai BD, Jalangaonkar VN, Mule RS. Record of pests infesting arecanut (Areca catechu Linneaus) and their seasonal incidence in Konkan region of Maharashtra. Indian Journal of Arecanut, Spices and Medicinal Plants 2002;4:120-2.

37. Phillips JS. Immature nutfall of coconuts in the British Solomon Islands. Bulletin of Entomological Research 1940;31:295-316.

38. Froggatt JL. Promecotheca antiqua Wse. Leaf pest of coconuts. New Guinea Agricultural Gazette 1937;3:21-2.

39. Way MJ, Cammell ME, Bolton B, Kanagaratnam P. Ants (Hymenoptera: Formicidae) as egg predators of coconut pests, especially in relation to biological control of the coconut caterpillar, Opisina arenosella Walker (Lepidoptera: Xyloryctidae), in Sri Lanka. Bulletin of Entomological Research 1989;79:219-33.

40. Williams DJ. The Pseudococcidae of the Solomon Islands. Bulletin of the British Museum (Entomology) 1960;8:387-430.

41. Way MJ, Bolton B. Competition between ants for coconut palm nesting sites. Journal of Natural History 1997;31:439-55.
42. Simmonds FJ. Biological control of Cordia curassavica (Boraginaceae) in Malaysia. Entomophaga 1980;25:363-4.

43. Fiedler K, Maschwitz $U$. The symbiosis between the weaver ant, Oecophylla smaragdina, and Anthene emolus, an obligate myrmecophilous lycaenid butterfly. Journal of Natural History 1989;23:833-46.

44. Hill DS. Agricultural Pests of the Tropics and their Control. 2nd ed. Cambridge University Press, Cambridge; 1983.

45. Dodd FP. Notes on the Queensland green tree ants (Oecophylla smaragdina). Victorian Naturalist 1902; 18:136-40.

46. Mahdihassan S. Mixed infection of lac. The Journal of the Bombay Natural History Society 1976;74:551-2.

47. Anon. Scale insects, mealybugs and lac insects. Planter's Bulletin of the Rubber Research Institute of Malaysia 1968;98:146-52.

48. Le Pelley RH. An oriental mealybug (Pseudococcus lilacinus Ckll.) and its insect enemies. Transactions of the Royal Entomological Society of London 1943;93:73-93.

49. Valentine P. Australian Tropical Butterflies. Frith and Frith, Queensland, Australia; 1988.

50. Ballmer GR. Observations on resource partitioning among ants (Hymenoptera: Formicidae) and lycaenid larvae (Lepidoptera: Lycaenidae) associated with Pueraria phaseoloides in South Thailand. ScienceAsia 2003;29: 197-202.

51. Green EE. Remarks on Coceidae collected by Mr Edward Jacobson, of Samarang, Java, with descriptions of two new species. Tijdschrift voor Entomologie 1913;55:311-8.

52. Macnae W. A general account of fauna and flora of mangrove swamps and forests in Indo-West-Pacific Region. Advances in Marine Biology 1968;6:73-270.

53. Tan R. Mangrove and Wetland Wildlife at Sungei Buloh Wetlands Reserve: Weaver Ants. 2001. Available from: URL: http://www.naturia.per.sg/buloh/inverts/weaver_ants.htm (accessed 14 October 2006).

54. Jesudasan RW, Claver MA, Priyakumari CJ, Ragupathy E. Some ethological observations in whitefly and ant interaction. Journal of Applied Zoological Researches 2004;15:17-21.

55. Khoo SG. Hypsipyla shoot borers of Meliaceae in Malaysia. In: Floyd RB, Hauxwell C, editors. Proceedings of an International Workshop on Hypsipyla Shoot Borers in Meliaceae. ACIAR, Canberra; 2001. p. 24-30.

56. Quick WNB. Early stages of the life cycle of Narathura araxes eupolis (Miskin) (Lepidoptera: Lycaenidae). The Victorian Entomologist 1974;4:24

57. Jinda L. The weaver ants, Oecophylla longinoda, Oecophylla smaragdina, Malaysia. Nature Malaysiana 1982;7:16-17.

58. Benzie JAH. Selective positioning of arboreal tents by weaver ants Oecophylla smaragdina (F.): A possible co-evolutionary development with maha-dan trees, Syzygium cumini (L.) Skeeis. Australian Entomological Magazine 1985;12:17-19.

59. Chantaranothai $P$, Parnell JAN. The breeding biology of some Thai Syzygium species. Tropical Ecology 1994;35:199-208.

60. Balakrishnan MM, Vinodkumar PK, Prakasan CB. A note of green scale-ant association on coffee. Indian Coffee, Bulletin of the Indian Coffee Board 1992;56:5-6. 
61. Miller NCE. Coccus (Lecanium) viridis Green: the 'Green scale' of coffee. In: Federated Malay States Government Press, Malaya (Malaysia); 1931. p. 17-29.

62. Van Mele P, Cuc NTT, Van Huis A. Direct and indirect influences of the weaver ant Oecophylla smaragdina on citrus farmers' pest perceptions and management practices in the Mekong Delta, Vietnam. International Journal of Pest Management 2002;48:225-32

63. Greenslade PJM. Phenology of three ant species in the Solomon Islands. Journal of the Australian Entomological Society 1971;10:241-52.

64. Leu AF. Organic lychee and rambutan production. In: Chomchalow N, Sukhvibul N, editors. Proceedings of the Second International Symposium on Lychee, Longan, Rambutan and Other Sapindaceae Plants. ISHS, Thailand; 2005. p. 241-54.

65. Tsuji K, Hasyim A, Harlion, K. Nakamura. Asian weaver ants, Oecophylla smaragdina, and their repelling of pollinators. Ecological Research 2004;19:669-73.

66. Das GM. Observations on the association of ants with coccids of tea. Bulletin of Entomological Research 1959;50:437-48.

67. Djieto-Lordon C, Dejean A. Tropical arboreal ant mosaics: innate attraction and imprinting determine nest site selection in dominant ants. Behavioral Ecology and Sociobiology 1999;45:219-25.

68. Hölldobler B. Territories of the African weaver ant. Zeitschrift fur Tierpsychologie 1979;51:201-13.

69. Strickland AH. The entomology of swollen shoot of cacao. II. The bionomics and ecology of the species involved. Bulletin of Entomological Research 1951;42:65-103.

70. Pierce NE, Braby MF, Heath A, Lohman DJ, Mathew J, Rand DB, et al. The ecology and evolution of ant associations in the Lycaenidae (Lepidoptera). Annual Review of Entomology 2002;47:733-71.

71. Bishop S. Pest Risk Analysis for Cerataphis lataniae Boisduval. Central Science Laboratory, York, UK; 2005. Available from: URL: http://www.defra.gov.uk/planth/pra/ Ceratap.pdf (accessed 1 December 2007).

72. Butani DK. Insect pests of fruit crops and their control: jackfruit. Pesticides 1979;13:36-40.

73. Way MJ. Studies on the association of the ant Oecophylla Ionginoda (Latr.) with the scale insect Saissetia zanzibarensis Williams. Bulletin of Entomological Research 1954;45:113-34.

74. Van Mele P. Mission de prospection sur les fourmis rouges dans les vergers de mangues biologiques et développement des activités pour découvrir en pratiquant. FAO, Rome; 2007.

75. Van Mele P. A historical review of research on the weaver ant Oecophylla in biological control. Agricultural and Forest Entomology 2008;10:13-22.

76. Van Mele P. Evaluating Farmers' Knowledge, Perceptions and Practices: A Case Study of Pest Management by Fruit Farmers in the Mekong Delta, Vietnam. Wagenigen University, The Netherlands; 2000.

77. Van Mele P, Chien HV. Farmers, biodiversity and plant protection: developing a learning environment for sustainable tree cropping systems. Journal of Agricultural Sustainability 2004;2:67-76.

78. Peng RK, Christian K. The weaver ant, Oecophylla smaragdina (Hymenoptera: Formicidae), an effective biological control agent of the red-banded thrips, Selenothrips rubrocinctus (Thysanoptera: Thripidae) in mango crops in the Northern Territory of Australia. International Journal of Pest Management 2004;50:107-14. 